\title{
Foot Digit 5 Phalanx
}

National Cancer Institute

\section{Source}

National Cancer Institute. Foot Digit 5 Phalanx. NCI Thesaurus. Code C52782.

A bone in the smallest and most lateral toe of the foot. 